\title{
Editorial for the Fourth International Conference on Energy-Aware High Performance Computing
}

\author{
Wolfgang E. Nagel • Thomas Ludwig • Matthias Müller
}

Published online: 25 July 2013

(C) Springer-Verlag Berlin Heidelberg 2013

Titan, a Cray XK7 system installed at the Oak Ridge National Laboratory ranks first in the November 2012's TOP500 list and achieves an impressive 17.59 Petaflops on the Linpack benchmark using 560,640 cores. Such an amount of performance comes with a price: The power input of about $8 \mathrm{MW}$ would result in an annual electricity bill of more than 8 million Euros based on German price levels. At the same time, the US Department of Energy publishes roadmaps in its Exascale Computing Initiative and puts $20 \mathrm{MW}$ as a "practical power limit" for a future Exaflop computer by the end of this decade. Thus, we are more than a factor of 25 away from our performance goal but today's technology uses almost half of the acceptable power target.

This situation calls for an intensified research in the field of energy efficiency technology, also called Green IT which has found its way into High Performance Computing. Only a few years ago, the community considered HPC as the Formula 1 of computing and ignored the fact of dramatically rising operational costs. However, as with these race cars, we conceived means to reduce power consumption and at

\section{W.E. Nagel (凶)}

Technische Universität Dresden, Zentrum für Informationsdienste und Hochleistungsrechnen (ZIH), 01062 Dresden, Germany e-mail: wolfgang.nagel@tu-dresden.de

T. Ludwig

Scientific Computing, Wissenschaftliches Rechnen, Universität Hamburg, 20146 Hamburg, Germany

e-mail: ludwig@dkrz.de

\section{Müller}

Lehrstuhl für Hochleistungsrechnen und Rechen- und

Kommunikationszentrum, RWTH Aachen University, 52074

Aachen, Germany

e-mail: mueller@rz.rwth-aachen.de the same time increase the speed. HPC is also beginning to learn from the field of embedded systems where batterypowered hardware requires special mechanisms to reduce power consumption during phases of low performance demand.

In 2010 we started the EnA-HPC conference series in order to bring researchers, vendors, and HPC center administrators together. Its purpose is to foster discussions regarding the status and future of energy awareness in high performance computing.

Fields of interest cover all abstraction layers, from the lowest level of hardware technology, via operating system, compiler and application issues to facility technologies like air conditioning, sensor technology and heat reuse. A comprehensive effort at all these levels is necessary to yield the overall energy reduction required to enable Exaflop computers to be operational by the end of this decade - as predicted by the TOP500 list.

For this fourth edition of our conference on EnergyAware High Performance Computing the submissions cover several of the abstraction levels mentioned above and thus represent world-wide research efforts in high performance computing: hardware evaluation and modeling, application analysis, energy efficiency improvements, and facility optimizations. This variety guarantees a good coverage of crucial research questions. The ideas presented will trigger inspiring discussion during the event. We hope to contribute to a successful cooperation between vendors and users of HPC equipment and foster more research in this field of green HPC.

The organizers express their gratitude to all contributors to this journal. Their research efforts will render High Performance Computing economically and ecologically sustainable. We thank the program committee members as well 
as all reviewers for their efforts in selecting an attractive content for this journal and for the conference.

Dresden, September 2013

Wolfgang E. Nagel, Thomas Ludwig, Matthias Müller

Program committee members:

- Cosimo Anglano, Universita del Piemonte Orientale, Alessandria, Italy

- Costas Bekas, IBM Research Zurich, Rueschlikon, Switzerland

- Dzmitry Kliazovich, University of Luxembourg, Luxembourg

- Enrique S. Quintana-Orti, Jaume I University, Castellón de la Plana, Spain

- Francisco Javier García Blas, Universidad Carlos III de Madrid, Madrid, Spain
- Jean-Marc Pierson, University Paul Sabatier, Toulouse, France

- Julita Corbalan, Barcelona Supercomputing Centre, Barcelona, Spain

- Kalyan Kumaran, Argonne National Laboratory, Lemont, IL, USA

- Laurent Lefevre, INRIA, University of Lyon, Lyon, France

- Natalie Bates, Lawrence Berkeley National Laboratory, Berkeley, CA, USA

- Simon McIntosh-Smith, University of Bristol, Great Britain

- Vincent Heuveline, Karlsruhe Institute of Technology, Karlsruhe, Germany

- William Barton Sawyer, Swiss Center for Scientific Computing, Lugano, Switzerland 